\title{
Technology Analysis of Hot Pushing Pipe Bending and Horn Mandrel Design
}

\author{
Lili Huang ${ }^{1,2, a}$, Xiaoyang $\mathrm{Lu}^{1, \mathrm{~b}}$ \\ ${ }^{1}$ Institute of Engineering Mechanics, Shandong Jianzhu University, Jinan 250101, China \\ ${ }^{2}$ School of Materials Science and Engineering, Shandong Jianzhu University, Jinan 250101, China \\ ahuang0539@hotmail.com, 'by5504@yahoo.cn
}

Keywords: Hot pushing pipe bending, horn mandrel, elbow pipe, Archimedes spiral cord.

\begin{abstract}
The forming principle and technological process of hot pushing pipe bending were introduced in this paper. Design of horn mandrel which affecting the elbow size and shape was studied. The push bending forming depends on the geometrical shape of horn mandrel. Two main design methods of horn mandrel were given and improvements have been made. The improved horn mandrel can push out high quality elbow pipe, which lay a good foundation for more accurate mandrel design and more rational process formulation.
\end{abstract}

\section{Introduction}

As a weak link in piping system, elbow pipes have close relations to safety operation of the whole piping system. Therefore, structural design of elbow pipe is very important[1]. Of various pipe forming technology, hot pushing pipe bending process is the most economic efficacious one. The elbow is formed by continuously pushing through an ox-horned mandrel with medium frequency induction heating. When using traditional bending process for forming, the wall thickness of the convex side is thinned or cracked for tension and the wall thickness of the concave side is thickened or wrinkled for compression. Hot pushing pipe bending technology can avoid these defects to a certain extent[2]. At present, this technology is widely used in gas and liquid conveying system and metal component. Many scholars have carried out theoretical analysis and experimental research $[3,4]$. Some basic theoretical problems in the forming process, such as, the force and deformation characteristics, the rule of metal flowing and the mechanism of plastic deformation, are still need further exploration and improving[5,6]. This paper introduces the forming principle and technological process of hot pushing pipe bending and studies the design of horn mandrel which is the key technology parameters in the forming process. It will play a positive role in revealing the pipe forming mechanism.

\section{Technological process of hot pushing pipe bending}

The hot pushing pipe bending process use continuous pushing and bending method on ox-horned mandrel with medium frequency induction heating, and then straight pipe billet is pushed and bended to elbow pipe in sequence. Its technological process is show in Fig.1.

\subsection{Technological process}

(1) A certain length and diameter of pipe billets are selected and set on straight draw bar (Part I as seen in Fig.1). The front end of the draw bar is welded to horn mandrel and the back end of it is linked to pushing machine with bearing lock and nonbearing lock.

(2) Pipe billets are pushing forward along the draw bar by thrust ring. The thrust ring is fixed on moved cross beam and driven by hydraulic motor. The thrust ring presses against the last one of pipe billet and pushes all the pipe billets into guide zone of horn mandrel (Part II as seen in Fig.1).

(3) When the moment of pipe billet entering into the bending and expanding zone (Part III as seen in Fig.1), medium frequency induction heating ring is heated to $700 \sim 900{ }^{\circ} \mathrm{C}$ and the bending deformation and expanding deformation occur at the same time in the pushing process. 
(4) After passing through the deformation zone of horn mandrel, the pipe enters into the shaping zone for shaping (Part IV as seen in Fig.1). The elbow pipe is pushed out from the large end of horn mandrel in sequence after shaping.

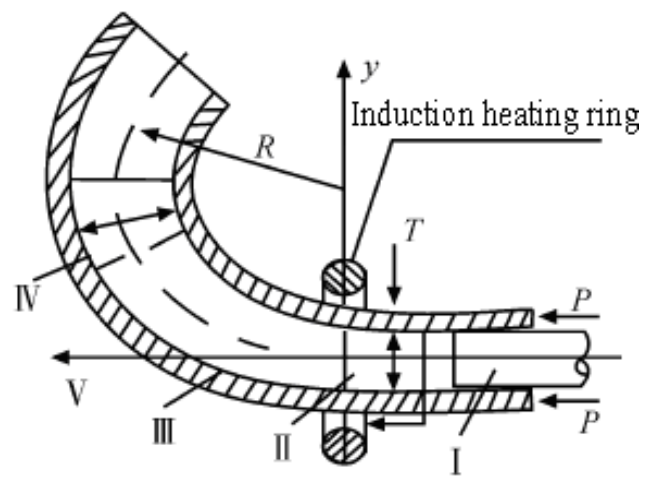

Fig.1 Schematic diagram of hot pushing pipe bending process

\subsection{Technological characteristics}

(1) This process can avoid the uneven thickness of traditionally formed bending pipes which is induced by the thickness reduction of the convex side under tensile stress and the thickness increase of the concave side under compressive stress.

(2) Minor radius, thin-wall and $180^{\circ}$ elbows with even wall thickness can be pushed out by using this process, and that can not be achieved by using other elbow forming process.

(3) If all of the technological parameters are set reasonable, this process can guarantee that all parts of the elbow wall thickness remain unchanged in the forming process and equal to the pipe billet wall thickness before deformation.

(4) Multiple bending angles of elbow with the same diameter, such as $45^{\circ}, 90^{\circ}$ and $180^{\circ}$, can be pushed out in one time on the same horn mandrel by this process. The production efficiency is high.

When the straight pipe billet cling to the horn mandrel for forming at high temperature, the bending deformation and expanding deformation is occurred at the same time. From mechanics point of view, the forming process of hot pushing pipe bending is a dynamic contact problem which force and displacement boundary condition are in changing and both material and geometric are nonlinear. There are many factors influencing the forming quality of elbow, such as the shape size of horn mandrel, heating temperature, pushing speed and pipe billet dimension, etc. These factors are correlated with each other in the forming process and bring great difficulties in experimental study and theoretical analysis.

\section{Design of horn mandrel}

Design of horn mandrel is more critical in elbow pipe production. The push bending forming depends entirely on the geometrical shape of horn mandrel. At present, there are four main ways to determine the shape and geometric dimension of horn mandrel. The first method is to select or modify existing data according to references and production practice. The second is to design by using experience. The third is to design by using theoretical analytical method. The fourth is to modify the theoretical design by using production data.

\subsection{Determination of pipe billet}

The diameter of pipe billet should be selected before design of horn mandrel. Table 1 shows the parameters of pipe billet. After computing the pipe billet parameters, close pipe billet is selected according to seamless steel pipe national standard specification table.

The given geometric dimensions of elbow pipe are as follows: the nominal diameter $D_{N}=150 \mathrm{~mm}$, the outer diameter $D_{w}=168 \mathrm{~mm}$, the bending radius $R=225 \mathrm{~mm}$, the wall thickness $s=6 \mathrm{~mm}$. According to the calculation formula of pipe billet, the outer diameter of the pipe billet $D_{P}=124 \mathrm{~mm}$, the length of the pipe billet $L_{P}=523.98953 \mathrm{~mm}$ and the wall thickness of pipe billet $S_{P}$ is $6 \mathrm{~mm}$. 
Table 1 Parameters of pipe billet

\begin{tabular}{|c|c|c|}
\hline Parameter & Calculation equation & Instruction \\
\hline $\begin{array}{c}\text { Outer } \\
\text { diameter } D_{P}\end{array}$ & $D_{P}=\left(D_{w}-S\right) /\left(1+D_{w} /(2 R)\right)+S$ & $\begin{array}{l}\text { If no appropriate specification, close or bigger } \\
\text { billet can be selected }\end{array}$ \\
\hline Length $L_{P}$ & $L_{P}=D_{w} \times R \times \theta / D_{P}+0.3 D_{N}$ & $\Delta L=0.3 D_{N}$ is working allowance \\
\hline $\begin{array}{l}\text { Wall thickness } \\
\qquad S_{P}\end{array}$ & $S_{P}=S$ & $\begin{array}{l}\text { It should be consistent with the thickness of } \\
\text { elbow pipe }\end{array}$ \\
\hline
\end{tabular}

Note: $D_{\mathrm{w}}$ is outer diameter of elbow pipe, $R$ is bending radius, $S$ is wall thickness of elbow pipe, $\theta$ is bending angle (radian), $D_{\mathrm{N}}$ is nominal diameter of elbow pipe.

\subsection{Design of horn mandrel}

The horn mandrel can be divided into three sections of guiding section, expanding deformation section and shaping section.

The first design method. The inner diameter of pipe billet $d_{1}=D_{P}-2 S_{P}$. The guiding section is a straight bar and its outer diameter slightly less than the inner diameter of pipe billet. It plays a role of guiding the pipe billet and transferring the pushing force.

The maximum diameter at the end of expanding deformation section is $d_{2}=D_{w}-2 S_{P}$. The bending radius of external arc on the expanding deformation section is $R_{1}=R+d_{2} / 2$. The bending radius of internal arc on the expanding deformation section is $R_{2}=\left(R-d_{2} / 2\right)+2\left(d_{2}-d_{1}\right) / \sin 45^{\circ}$.

The bending radius of external arc on the shaping is $R_{3}=R+d_{2} / 2$. The bending radius of internal arc on the shaping section is $R_{4}=R-d_{2} / 2$.

The second design method. Because the curvature radius of elbow pipe is consistent with that of horn mandrel, Archimedes spiral cord can be used to design the axis of horn mandrel and it can ensure that the expanding deformation section bend smoothly. The external dimensions of horn mandrel are related to Archimedes coefficient $a$, bending angle $\theta$ and expanding ratio $K$.

The formula of Archimedes spiral cord in polar coordinates is $r=\alpha \theta$. The curvature formula in polar coordinates is shown as Eq.(1) and the radius of curvature is shown as Eq.(2).

$$
\begin{aligned}
& K=\frac{\left|r^{2}+2 r^{\prime 2}-r r^{\prime \prime}\right|}{\left(r^{2}+r^{\prime 2}\right)^{3 / 2}}=\frac{\theta^{2}+2}{\alpha\left(\sqrt{\theta^{2}+1}\right)^{3}} \\
& R=\frac{1}{K}=\frac{\alpha\left(\sqrt{\theta^{2}+1}\right)^{3}}{\theta^{2}+2}
\end{aligned}
$$

The bending angle $\theta$ is obtained by substituting the axis bending radius of elbow pipe and Archimedes coefficient $a$ into the formula of curvature radius. The intersection position of expanding section and shaping section can be determined by calculating the curvature radius of Archimedes spiral cord at this angle position $\theta$. The axis shape is determined after the bending angles of expanding section and shaping section are selected. The axis of expanding section which uses Archimedes spiral cord is tangent to the axis of shaping section which equals to the bending radius of elbow pipe axis.

Based on the above design method, the horn mandrel can be improved as follows. A conical expanding part is added between the guiding section and expanding deformation section. This part makes the pipe billet hoop on the horn mandrel tighter and tighter before entering the expanding deformation section and occurring plastic deformation. The design of the conical expanding part requires that the pipe billet only deforms elastically at this stage. The designed horn mandrel by using three-dimensional modeling software UG is shown in Fig. 2. 


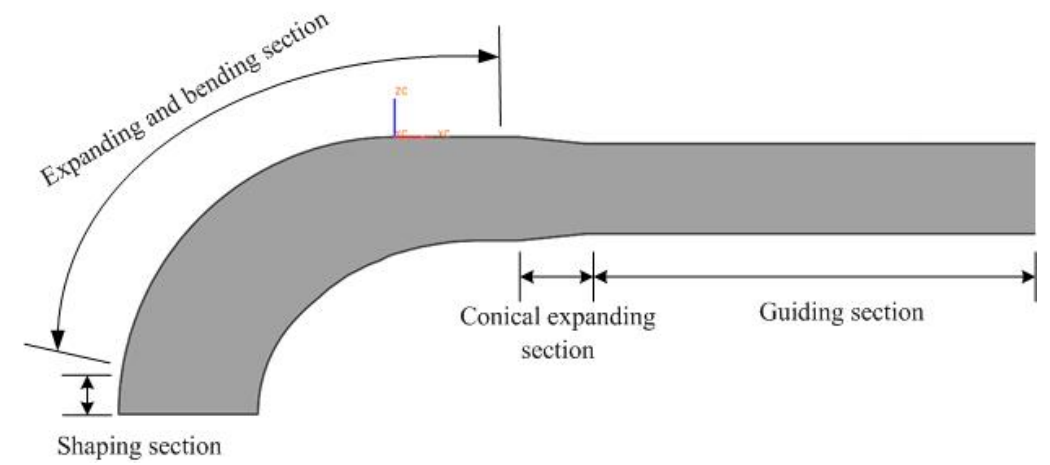

Fig.2 Three dimensional modeling of horn mandrel

\section{Summary}

By analyzing the forming principle and technological process of hot pushing pipe bending, two main design methods of horn mandrel are given. Based on the two design methods, improved design method of the horn mandrel is proposed. Proved by practice, the improved horn mandrel can push out elbow pipes with high quality and good mechanical properties, which will lay a good foundation for more accurate mandrel design and more rational process formulation.

\section{Acknowledgements}

This research was supported by Foundation for Outstanding Young Scientist in Shandong Province (BS2013ZZ015) and National Natural Science Foundation of China (11272188).

\section{References}

[1] Xiaoyang Lu, Kui Li, Bingye Xu, et al. Medium frequency hot pushing pipe bending process and forming mechanism study. Forging \& Stamping Technology. (1994) No. 4, p. 11-14.

[2] Tiande Miao, Renji Tang, Jianguo Zhang. A plastic analysis for forming complex deformation bent tubes by pushing them through a horn-like axle-tree. Chinese Journal of Theoretical and Applied Mechanics. Vol. 3 (1979), p. 230-238.

[3] Xiaoyang Lu, Bingye Xu, Liping Lu, et al. Application of fuzzy stein optimal design of the process parameters of medium frequency induction heated pushing bending tubes. Journal of Plasticity Engineering. (1994) No. 4, p. 11-18.

[4] Zhenyi Jiang, Xianghua Liu, Guodong Wang. Forming Processes of stainless steel pipe bend with large diameter and thick wall. Journal of Plasticity Engineering. (1997) No. 4, p. 65-69.

[5] Yu Ding, Peifen Weng. Numerical Study of Theoretical Model and Flow Characteristic in $90^{\circ}$ Elbow Pipe. Chinese Journal of Computational Mechanics. Vol. 6 (2004), p. 314-315.

[6] Xiaoyang Lu, Qitao Zhou, Lili Huang, et al. Numerical simulation and parameter optimization of hot pushing elbow pipe bending process. Applied Mechanics and Materials. Vol. 432 (2013), p. 92-97. 